\title{
Efficacy of Cannabinoids on Spasticity and Chronic Pain in a Patient with Co-occurrence of Multiple Sclerosis and Neurofibromatosis Type 1
}

\author{
Eleonora Virgilio, Domizia Vecchio, Paola Naldi, Roberto Cantello \\ Neurology Unit, Maggiore della Carità Hospital, Department of Translational Medicine, University of Piemonte Orientale, Novara, Italy
}

Received: $26 / 02 / 2021$

Accepted: $25 / 04 / 2021$

Published: $13 / 05 / 2021$

How to cite this article: Virgilio E, Vecchio D, Naldi P, Cantello C. Efficacy of cannabinoids on spasticity and chronic pain in a patient with co-occurrence of multiple sclerosis and neurofibromatosis 1. EJCRIM 2021;8: doi:10.12890/2021_002424.

Conflicts of Interests: The authors declare there are no competing interests.

This article is licensed under a Commons Attribution Non-Commercial 4.0 License

\section{ABSTRACT}

Neurofibromatosis type 1 (NF1) is a rare autosomal dominant disease involving the skin and central nervous system (CNS), and also characterized by skeletal and spinal schwannomas that may cause chronic neurogenic pain. Furthermore, pain in NF1 is underestimated, even though it has an impact on quality of life. Multiple sclerosis (MS) is the most common acquired demyelinating disease that may in later stages present with refractory spasticity, particularly in the lower limbs. Oromucosal cannabinoid sprays are currently available for spasticity treatment in MS, with encouraging results on MS pain, but few data have been reported regarding the use of cannabinoids in NF1. We report the successful treatment of chronic neurogenic pain and spasticity in a patient with co-occurrence of NF1 and MS after a poor response to standard approaches.

\section{LEARNING POINTS}

- Chronic pain is a possible complication of several neurological conditions and may show a poor response to standard drugs, thus affecting quality of life.

- Oromucosal cannabinoid sprays are routinely used in multiple sclerosis spasticity.

- Cannabinoids may be also effective against neurogenic pain in neurofibromatosis type 1

\section{KEYWORDS}

Multiple sclerosis, neurofibromatosis type 1, spasticity, chronic pain, cannabinoids

\section{CASE DESCRIPTION}

A Caucasian man with a family history of neurofibromatosis type 1 (NF1) (his father, sister and brother were affected) was himself diagnosed because of multiple cutaneous and subcutaneous neurofibromas and café-au-lait spots. Genetic testing confirmed the diagnosis when he was 30 years of age. At the age of 45 , he was also diagnosed with medullary thyroid cancer and at the age of 56 with a gastric neuroendocrine tumour, both of which were successfully surgically removed. He was first evaluated in our multiple sclerosis (MS) centre at the age of 55 for progressive sensory-motor paraparesis with hyperreflexia, numbness, paraesthesia, neurogenic pain and bladder dysfunction with onset 1 year previously. He reported he had previously experienced sensory and motor symptoms with remissions in his third decade of life but did not undergo any neurological evaluation at the time. 
Brain and spinal magnetic resonance imaging (MRI) revealed multiple T2 and FLAIR-weighted white matter, corpus callosum lesions, and a C3 cervical lesion suggestive for MS. MRI findings were not typical for unidentified bright objects (UBO) normally found in NF1 (Fig. 1). Blood tests for autoimmune and infectious conditions were unremarkable and cerebrospinal fluid (CSF) analysis showed oligoclonal bands. Based on history, examination and MRI results, we concluded the patient had MS according to the 2010 McDonald diagnostic criteria ${ }^{[1]}$. One year later, spinal MRI showed two new dorsal demyelinating lesions without contrast enhancement; the exam excluded spinal neurofibromas and showed initial degenerative discopathy at C3-C7 levels (Figs. 2 and 3). Clinically, the patient's neurological deficits, particularly the pyramidal involvement, improved over time, but he still needed unilateral assistance with muscular spasms when walking long distances. He also complained of sensory paraesthesia, and constant chronic pain with dysesthesia in the lower limbs particularly the right limb, even when resting (Visual Analogue Scale (VAS): 7/10). The patient progressively developed spasticity particularly in the lower limb extensor muscles (Modified Ashworth Scale (MAS): 13/48).
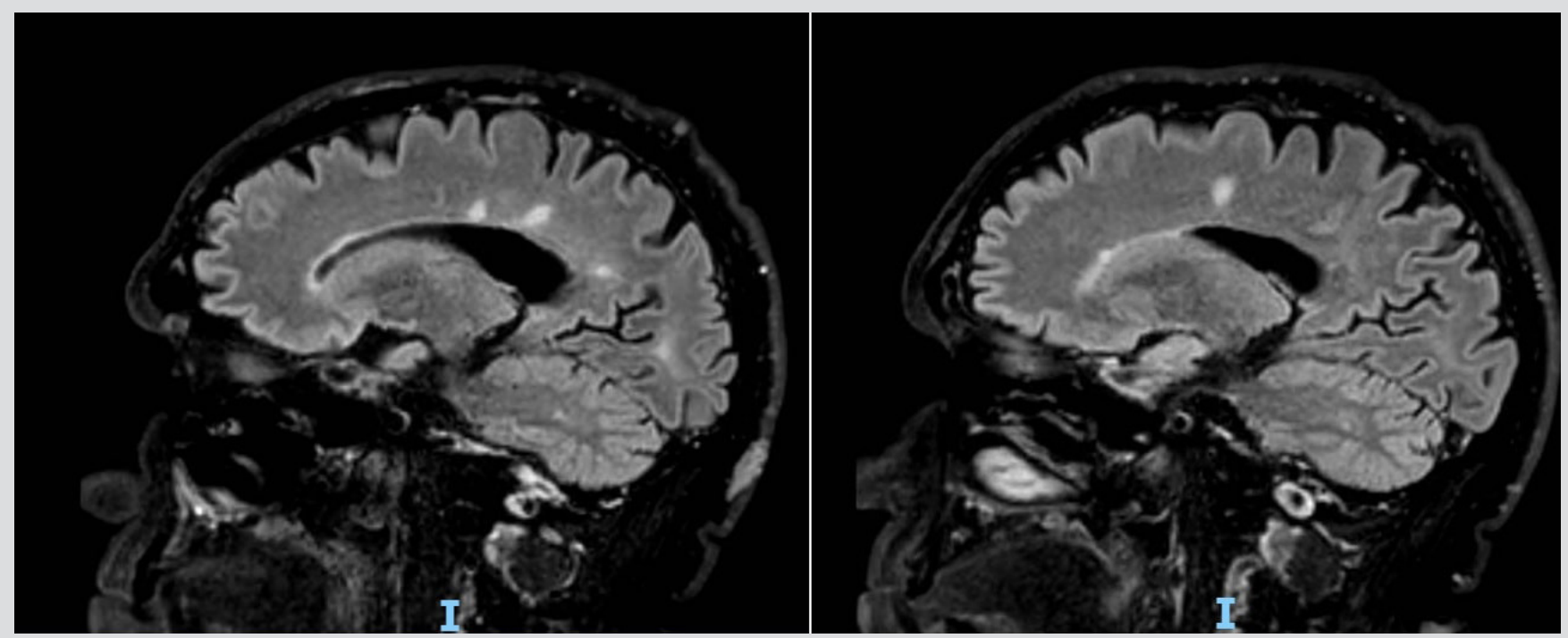

Figure 1. Sagittal brain MRI showing multiple white matter inflammatory lesions and subcutaneous neurofibromas
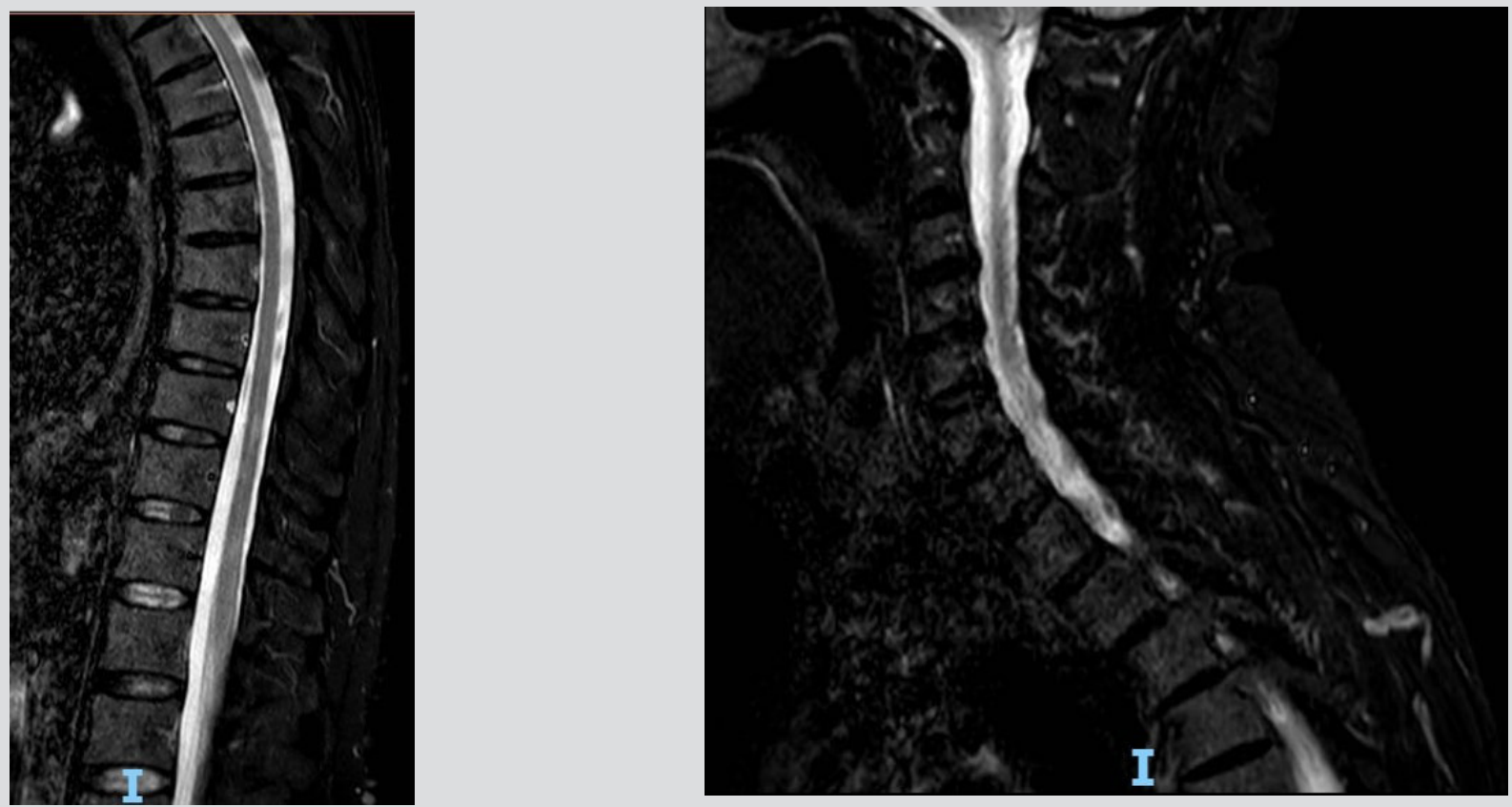

Figure 2. Sagittal spinal MRI showing two new dorsal inflammatory lesions

Figure 3. Sagittal spinal MRI showing diffuse cervical discopathy 
At this point, he was moderately disabled, with an EDSS of 4.5/10. Immunomodulatory disease-modifying treatment was postponed in light of the new diagnosis of neuroendocrine gastric cancer. He was treated with baclofen up to $25 \mathrm{mg} / \mathrm{daily}$ (considered the first-line treatment for MS spasticity) with severe side-effects, particularly low blood pressure. Several different standard treatments for neuropathic pain (carbamazepine, gabapentin, pregabalin, duloxetine, venlafaxine, amitriptyline) were administered with poor results on sensory manifestations and frequent side-effects. Finally, an oromucosal cannabinoid spray was started to treat spasticity and resulted in subjective and objective reduction in hypertonia (MAS 6/48) and in neuropathic pain and dysesthesia (VAS 2/10), and improvement in quality of life and walking independence.

\section{DISCUSSION}

Chronic pain may be part of the clinical manifestations of several neurological diseases, presenting with high variability in type and intensity and difficulty in management. The pathophysiology of pain in MS includes pain directly related to the brain and spinal inflammatory lesions, with central pain due to corticospinal system disinhibition and/or chronic activation of nociceptive afferents. Pain may also be secondary to complications of the disease, such as spasticity and contractures, or as a side effect of chronic treatment (i.e., steroids, interferon beta, glatiramer acetate) ${ }^{[2]}$. On the other hand, NF1 patients may experience pain due to scoliosis and skeletal alterations, or secondary to spinal schwannomas or other tumours such as abdominal neoplasms ${ }^{[2-4]}$. Moreover, a high percentage of individuals with NF1 report significant daily chronic pain and diffuse discomfort, often not localized to a specific structural lesion or abnormalities, presenting treatment challenges for the clinician ${ }^{[2,3]}$.

At the moment, pain in NF1 patients is probably an underestimated problem. The pathophysiology has not yet been completely elucidated ${ }^{[3,4]}$, but an American survey of 255 NF1 patients showed a high prevalence of chronic pain particularly in patients undergoing tumour removal surgery, but with the pain not necessarily being located in the area of surgery, and only $7 \%$ of participants having plexiform tumours ${ }^{[2]}$. The areas most affected with pain were the limbs, particularly the arms ${ }^{[2]}$. There are few reports on the use of pharmacological treatment for NF1, with opioids being the most used ${ }^{[2,3]}$ and only one study reporting one single patient with NF1 treated with cannabinoids ${ }^{[5]}$, although the use of cannabinoids in cancer patients is currently a hot topic. Several scales and patient- reported outcomes (i.e., VAS) may be used to assess chronic pain, and are particularly recommended in both clinical practice and research contexts to determine symptom variation over time ${ }^{[2]}$. The MAS scale is currently the most used clinical tool to test spasticity.

Several pharmacological treatmentsareavailableforpain, withcannabinoidsalsocurrentlyavailableforMSpatients, withefficacyonspasticity, pain and bladder dysfunction ${ }^{[2]}$. The current oromucosal formulation contains the psychoactive component $\Delta 9$-tetrahydrocannabinol (THC) and the non-psychoactive component cannabidiol (CBD) in a ratio of 1:1, both acting on the endocannabinoid system in the CNS. We have described the case of a patient experiencing chronic neuropathic pain which developed after MS disease progression, who also presented with NF1 with neoplastic comorbidities and cervical discopathy which are common features in NF1 patients. We hypothesize that both the MS and NF1 are responsible for the lower limb neuropathic pain in our patient, but cannot exclude that MS is the principal cause. Nonetheless, we have shown that cannabinoids are also effective in NF1 patients and have reported successful treatment of both spasticity and chronic neuropathic pain, highlighting the possibility of nabiximol use in non-conventional neurological diseases such as NF1.

\section{REFERENCES}

1. Polman CH, Reingold SC, Banwell B, Clanet M, Cohen JA, Filippi M, et al. Diagnostic criteria for multiple sclerosis: 2010 revisions to the McDonald criteria. Ann Neurol 2011;69(2):292-302. doi: 10.1002/ana.22366. PMID: 21387374; PMCID: PMC3084507.

2. Buono FD, Grau LE, Sprong ME, Morford KL, Johnson KJ, Gutmann DH. Pain symptomology, functional impact, and treatment of people with neurofibromatosis type 1. J Pain Res 2019;12:2555-2561. doi: 10.2147/JPR.S209540. PMID: 31692483. PMCID: PMC6710538.

3. Shofty B, Barzilai O, Khashan M, Lidar Z, Constantini S. Spinal manifestations of neurofibromatosis type 1. Childs Nerv Syst 2020;36(10):2401-2408. doi: 10.1007/s00381020-04754-9. PMID: 32564155.

4. Aboud T, Schuster NM. Pain management in multiple sclerosis: a review of available treatment options. Curr Treat Options Neurol 2019;21(12):62. doi: 10.1007/s11940-0190601-2. PMID: 31773455

5. Wade DT, Robson P, House H, Makela P, Aram J. A preliminary controlled study to determine whether whole-plant cannabis extracts can improve intractable neurogenic symptoms. Clin Rehabil 2003;17(1):21-29. doi: 10.1191/0269215503cr581oa. PMID: 12617376. 